\title{
When Outliers Are Different
}

\author{
J. I. Katz, ${ }^{1 \star}$ \\ ${ }^{1}$ Department of Physics and McDonnell Center for the Space Sciences, Washington University, St. Louis, Mo. 63130 USA
}

2 September 2021

\begin{abstract}
When does the presence of an outlier in some measured property indicate that the outlying object differs qualitatively, rather than quantitatively, from other members of its apparent class? Historical astronomical examples include the many types of supernovæ and short vs. long Gamma Ray Bursts. A qualitative difference implies that some parameter has a characteristic scale, and hence its distribution cannot be a power law (that can have no such scale). If the distribution is a power law the objects differ only quantitatively. The applicability of a power law to an empirical distribution may be tested by comparing the most extreme member to its next-most extreme. The probability distribution of their ratio is calculated, and compared to data for stars, radio and X-ray sources, and the fluxes, fluences and rotation measures of Fast Radio Bursts (FRB). It is found with high statistical significance that the giant outburst of soft gamma repeater SGR 1806-20 differed qualitatively from its lesser outbursts and FRB 200428 differed qualitatively from other FRB (by location in the Galaxy), but that in some supernova remnant models of rotation measure FRB 121102 is not, statistically significantly, an outlier.
\end{abstract}

Key words: radio continuum, transients: fast radio bursts, methods: statistical

\section{INTRODUCTION}

A question often asked in astronomy is whether a group of objects, similar in some ways, may be subdivided into qualitatively distinct subclasses. For example, novæ, originally considered a single class of events, were first subdivided into Galactic novæ and supernovæ (not observed in our Galaxy since 1604, but recognized in the Andromeda Galaxy in 1885). Subsequently, supernovæ have been divided into an ever-multiplying botanic garden of types and subtypes. Gamma-Ray Bursts (GRB) were divided into (Galactic and Magellanic Cloud) Soft Gamma Repeaters and (extragalactic) GRB, and then GRB into short and long GRB (Kouveliotou et al. 1993) that were found to differ not only in duration but in physical origin. Qualitative divisions have been essential to understanding these phenomena, because no physical models have explained them all as single classes of events differing only quantitatively.

Similar questions have arisen with regard to Fast Radio Bursts (FRB). If they are all manifestations of the same processes, then any proposed mechanism must be consistent with every FRB observed (allowing for quantitative differences in parameters and viewing geometry), while if there are two or more qualitatively different mechanisms or source objects producing FRB this constraint is relaxed. An apparent excess of bright bursts over the power law distribution of sources uniformly but randomly distributed in space (the familiar $N \propto S^{-3 / 2}$ relation of extragalactic radio astronomy, where $N$ is the cumulative number with flux greater than $S$ ) has

* E-mail katz@wuphys.wustl.edu been used (Katz 2017) to challenge the assumption of a statistically uniform spatial distribution, but this has been disputed (Macquart \& Ekers 2018) on the grounds of possible discovery bias of the very bright FRB 010724 .

More recently, the fluxes and fluences of many additional FRB, including homogeneous data sets from Parkes, UTMOST, ASKAP and CHIME/FRB, have been measured and the rotation measures (RM) of a number of FRB have also been obtained. Some of these datasets include outliers, and again raise the question of whether the outliers indicate the presence of two (or more) qualitatively different events classified as FRB, or of two qualitatively different environments of FRB (possibly, but not necessarily, related to different models of the FRB themselves).

In the simplest possible case, which arises in many extant data sets, there is a single outlier. When can we infer that it represents a different class of object or event? When there is only one outlier, cluster analysis is inapplicable.

The distribution of brightnesses of astronomical objects is a convolution of their intrinsic luminosities or energies with their spatial distribution, which is usually distributed over a very large range of distances. As a result, objects of distinct luminosity do not form distinct clusters of apparent brightness. For example, there are bright nearby dwarf stars and dim distant giant stars; even if stars were clustered in luminosity they would not be clustered in apparent brightness.

Each interval of intrinsic luminosity has a $N \propto S^{-3 / 2}$ distribution of observed flux or fluence $S$, as must the integral over their luminosity distribution. This only breaks down when a characteristic scale enters, such as the characteristic distance to the nearest member of the population (the 
$-1 / 3$ power of its number density), recently used by Lu, Beniamini \& Kumar (2021) to estimate the number density of sub-classes of FRB, or when the statistically homogeneous Euclidean distribution is cut off by the size of the Galaxy, the Local Group, other cosmic structure, or the Hubble distance. A related method (Wijers \& Van Paradijs 1991) has been used to infer the number of objects in a population from the total flux, the flux of the brightest member, and a power law fit to their luminosity function.

How extreme must an outlier be, in order that we may conclude, with statistical confidence, that it is qualitatively different from objects or events it otherwise resembles? This question arises even when only one parameter is studied and when as few as two objects have been observed.

Sec. 2 discusses the significance of power law distributions, not only in astronomy, but more generally. Sec. 3 asks if a single outlier in some variable is consistent with a power law distribution of that variable. This is motivated by the outlying flux and fluence of the discovery FRB 010724, the rotation measure of FRB 121102, the flux and fluence of FRB 200428 , and the giant outbursts of soft gamma repeaters (SGR). These objects or events are single outliers, not clusters of data; their consistency with a power law may be assessed by comparing them to the second-most outlying object or event. Sec. 4 applies this method to a number of classes of astronomical objects and events, while the Discussion (Sec. 5) summarizes these results and their implications.

\section{POWER LAWS}

It is a trivial observation, known at least since Kolmogorov's work on turbulence in the 1940's (Kolmogorov 1941; Tennekes 1972; Frisch 1995; Batchelor 1967), and discussed by Katz (1986) in the context of the model now called Self-Organized Criticality, that a power law distribution of a parameter as a function of an independent variable is inconsistent with the existence of a characteristic scale of that variable: A power law is a straight line on a log-log plot, any portion of which may be transposed to any other portion by scaling factors. Any deviation from a single power law defines a characteristic scale, the value of the independent variable at which the actual distribution deviates, perhaps by a change of the exponent in the power law.

Deviations from power laws may have many different origins. These may include selection biases. The most obvious example of such a bias results from the fact that more distant sources are fainter. If the sources are standard candles, there will be a cutoff distance beyond which none are detected. In this case, the deviation from the power law reflects their detectability, not any intrinsic property of the sources. In order to ensure that a deviation from a power law indicates an intrinsic property, such biases must be avoided.

If there is no characteristic scale, so that all objects in the class are qualitatively similar although quantitatively different, the observed distribution must be a power law. Hence consistency of the distribution with a power law is a test of the hypothesis that the objects cannot be divided into qualitatively different subclasses. Inconsistency with a power law demonstrates the existence of a qualitative distinction. An outlier, by definition, is inconsistent with the distribution observed of the other objects. The probability of this occurring by chance may be calculated as a function of the power law slope of the other elements of the class and of the degree to which the outlier is beyond the extrapolation of the observed distribution of the other objects. This is most simply quantified by comparing the value of the observed parameter of the most extreme (outlying) element to that of the secondmost extreme. If there are multiple outliers it will generally be evident that there are two distinct subclasses of objects, that can be separated by cluster analysis, but in some cases of interest there is only one outlier.

\section{SINGLE OUTLIERS AND FIRST/SECOND RATIO}

All power law distributions must have at least one cutoff or break in order that the total number of objects be finite. When the independent variable is flux, fluence, luminosity, or an analogous energetic parameter, the break must be sharp (between two power laws, their exponents must differ by $>1$ ) in order that both the number of objects and their integrated radiated power or energy be finite. The question is not whether the power law is broken but whether the break occurs within the observed range of the independent variable. If it does, then the break divides the observed objects into two qualitatively different classes. If not, then all the observed objects may be qualitatively similar, though quantitatively differing. Even so, they are not necessarily qualitatively similar, as shown by the examples of dwarf and giant stars, or main sequence and white dwarf stars, all of whose flux distributions are described by a $N \propto S^{-3 / 2}$ law because they are homogeneously distributed in a Euclidean space (up to cutoffs at the thickness of the Galactic disc and the distance of nearest neighbors).

If there are many outliers, by definition of "outlier" a break divides them from the remainder of the objects. It will generally be evident that they form a distinct distribution, often with other evidence of their difference (for example, supernovæ and novæ have very different spectra and temporal behavior, while long and short GRB differ in duration).

The problem addressed here is that of a single outlier. When can we say that there must be a break in the distribution between it and the object with the second-most extreme value of the independent variable, that observation of the outlier is inconsistent with a power law that describes the distribution of the independent variable over less extreme objects?

Suppose a power law differential number distribution as a function of an independent variable $x$

$\frac{d N}{d x}=C x^{-\gamma}$.

By definition, there is one object with $x>x_{2}$, where $x_{2}$ is the second-highest value of $x$. Then

$1=\int_{x_{2}}^{\infty} \frac{d N}{d x} d x=\frac{C}{\gamma-1} x_{2}^{-\gamma+1}$

and

$C=(\gamma-1) x_{2}^{1-\gamma}$.

The requirement that the total number of objects be finite, integrating as $x \rightarrow \infty$ implies $\gamma>1$. If $x$ represents an energy-like quantity (flux, fluence, luminosity, etc.), finiteness of the integral implies $\gamma>2$. In homogeneously filled three 
dimensional Euclidean space in the limit $x \rightarrow \infty \gamma=5 / 2$. The divergence

$\int S d N=\int S \frac{d N}{d S} d S \propto \int S^{-3 / 2} d S \propto S^{-1 / 2}$

as $S \rightarrow 0$ describes Olber's Paradox, resolved by a cutoff at large distance (Hubble radius, or size of the Galaxy) and small $S$. In two dimensional space, such as the Galactic disc, $\gamma=2$. In practice, $\gamma$ is found by fitting to the observed distribution for $x<x_{2}$.

The probability that the highest value of $x$ is as large or larger than the extreme outlier $x_{1}$ is

$P\left(x \geq x_{1}\right)=\int_{x_{1}}^{\infty} \frac{d N}{d x} d x=\left(\frac{x_{1}}{x_{2}}\right)^{1-\gamma}$.

An observed $x_{1}$ is inconsistent with an extrapolation of the power law at a confidence level $1-P$ if

$\frac{x_{1}}{x_{2}}>P^{1 /(1-\gamma)}$.

If $\gamma$ is known, inverting this expression shows the minimum value of $x_{1} / x_{2}$ required to reject, at a confidence level $1-P$, the hypothesis that the power law is unbroken between $x_{2}$ and $x_{1}$. Rejection implies that the outlier differs qualitatively from the remainder of the objects. If, as is often the case, $\gamma$ is not accurately determined, an observed value of $x_{1} / x_{2}$ sets a coupled constraint on the value of $\gamma$ and the confidence with which an unbroken power law can be rejected.

Fig. 1 shows $P$ as a function of $x_{1} / x_{2}$ for several values of $\gamma$. Fig. 2 shows $\gamma$ as a function of $x_{1} / x_{2}$ for several values of statistical significance $1-P$.

\section{APPLICATIONS}

The Table shows most extreme/second most extreme ratios for several astronomical datasets. Most of these objects are variable, so the values in the indicated catalogues are shown. This introduces uncertainty because there may be biases resulting from differences in observing methodology and the selection of catalogued values; for example, stronger variable sources may be observed more frequently and their greatest strengths recorded in the catalogue, biasing their catalogued strengths upward. Extreme examples of this are the maximum observed fluxes reported for transients. Some catalogues, such as those for specific FRB surveys, are likely homogeneous with minimal bias.

Objects distributed homogeneously in three dimensional space have $\gamma=5 / 2$, while Galactic disc objects, such as most entries in X-ray catalogues, are expected to have $\gamma=2$ because of the two dimensional geometry of a disc. These values of $\gamma$ assume no cutoff resulting from the cosmological redshift and the finite extent of the Galactic disc; these assumptions are tested here, and shown to be likely invalid for the AGN and $3 \mathrm{CR}$ (extragalactic) catalogues, and (with somewhat less significance) for the $4 \mathrm{U}$ catalogue.

Direct comparisons of the flux and fluence of FRB 200428 to those of other FRB are not quantitative because FRB 200428 does not come from a homogeneous catalogue. It is included here to show how its extreme outlying position is explained by its location in the Galaxy, a mass concentration inconsistent with a homogeneous Universe. The cosmological $\gamma=5 / 2$ cannot be extrapolated down to Galactic distances;

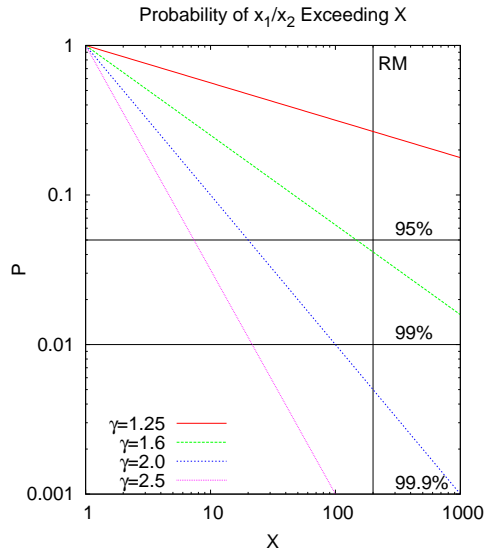

Figure 1. The probability that $x_{1} / x_{2}$ exceeds $X$ for several values of $\gamma$. Levels of statistical significance are indicated. $x_{1} / x_{2}=200$ is the first/second ratio of FRB Rotation Measure.

$\gamma=5 / 2$ is excluded with very high confidence. This is not a new discovery(!), but illustrates the method. It also lends confidence to the assertion that the Parkes catalogue of FRB does not provide compelling evidence that the discovery FRB 010724 is an outlier inconsistent with a statistically homogeneous cosmological distribution.

The 3CR catalogue (Bennett 1962a,b) is a classic catalogue of radio sources observed at $178 \mathrm{MHz}$. It is used here because its sources have been identified as Galactic (Spinrad et al. 1985) or extragalactic. Many entries in the $4 \mathrm{U}$ X-ray source catalogue (Forman et al. 1978) have not been identified, but are assigned as Galactic if $b \leq 20^{\circ}$. Fluences of SGR 1806-20 outbursts are from Hurley et al. (2005); Palmer et al. (2005); Golenetskii et al. (2007) with $\gamma$ from Gögüus et al. (2000); Götz et al. (2006) ( $x_{1}$ and $x_{2}$ are not from a homogeneous catalogue, introducing additional uncertainty). Crab pulsar Giant Pulses are from Bera \& Chengalur (2019). FRB are from the homogeneous catalogues in HeRTA/FRBSTATS (2021) except for CHIME from CHIME/FRB Collaboration (2021) and FRB 200428 from Bochenek et al. (2020); CHIME/FRB Collaboration (2020). FRB rotation measures (RM) are from Petroff et al. (2016) and Hilmarsson et al. (2021); the value of $\gamma$ is for the supernova remnant (SNR) model described in Sec. 5. FRB 121102 bursts are from a single homogeneous five hour sample with $x_{1} / x_{2}=1.55$, the average of the values of 


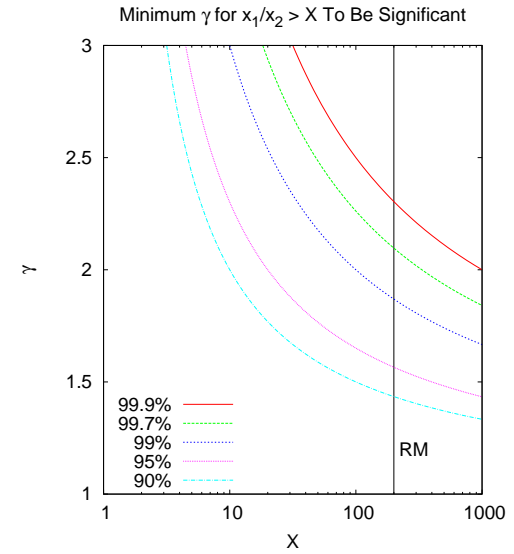

Figure 2. The the minimum value of $\gamma$ as a function of $X$ for which $x_{1} / x_{2}>X$ indicates that the outlier is inconsistent with an extrapolation of the power law, for the indicated levels of statistical confidence. $x_{1} / x_{2}=200$ is the first/second ration of FRB Rotation Measure.

Gajjar et al. (2018) (1.75) and Zhang et al. (2018) (1.35), but with $\gamma=1.7$ from Law et al. (2017); Wang \& Zhang (2019).

\section{DISCUSSION}

Table 1 shows a number of significant outliers, of which the most extreme is FRB 200428. This is explained by its presence in the Galaxy whose density (of mass, and likely of whatever astronomical objects make FRB) is orders of magnitude greater than the mean density of the local Universe. The extragalactic $\gamma=5 / 2$ does not allow for the Galactic density concentration. Because we are also located in the Galaxy, it is unsurprising that the one Galactic FRB should be an outlier in flux and fluence. This is not news, but confirms that the method finds the obvious.

More interesting are the facts that the AGN 3C273 is a significant outlier in the visible-light AGN population, Cyg A is a significant outlier in the $3 \mathrm{CR}$ extragalactic (radio galaxy) distribution and Sco X-1 is a significant outlier in the $4 \mathrm{U}$ Galactic $2-6 \mathrm{keV}$ X-ray source distribution. Perhaps these objects are fundamentally different from other members of their classes. The extragalactic FRB distributions contain no

\begin{tabular}{crccc}
\hline Parameter & $\mathrm{N}$ & $x_{1} / x_{2}$ & $\gamma$ & Significance \\
\hline Stars (V-band) & & 1.94 & $5 / 2$ & $63 \%$ \\
AGN (V-band) & & 10 & $5 / 2$ & $97 \%$ \\
3CR (extragalactic) & 298 & 8 & $5 / 2$ & $96 \%$ \\
3CR (Galactic) & 38 & 8 & 2 & $87 \%$ \\
4U (Galactic) & 181 & 18 & 2 & $94 \%$ \\
4U (transients) & 12 & 3.5 & 2 & $72 \%$ \\
SGR 1806-20 fluence & 760 & $7 \times 10^{4}$ & 1.7 & $99.96 \%$ \\
Crab Giant Pulses & 1100 & 1.25 & 2.8 & $33 \%$ \\
FRB Fluxes (Parkes) & 31 & 4.3 & $5 / 2$ & $89 \%$ \\
FRB Fluxes (UTMOST) & 15 & 1.37 & $5 / 2$ & $38 \%$ \\
FRB Fluxes (ASKAP) & 42 & 1.15 & $5 / 2$ & $19 \%$ \\
FRB Fluxes (CHIME) & 536 & 1.33 & 2.4 & $33 \%$ \\
FRB Fluences (Parkes) & 31 & 1.1 & $5 / 2$ & $17 \%$ \\
FRB Fluences (UTMOST) & 15 & 1.71 & $5 / 2$ & $55 \%$ \\
FRB Fluences (ASKAP) & 42 & 2.1 & $5 / 2$ & $67 \%$ \\
FRB Fluences (CHIME) & 536 & 1.0 & 2.4 & $0 \%$ \\
FRB 200428 Flux & & $1.7 \times 10^{4}$ & $5 / 2$ & $>99.9999 \%$ \\
FRB 200428 Fluence & & $3.6 \times 10^{3}$ & $5 / 2$ & $>99.999 \%$ \\
FRB RM & 19 & 200 & $5 / 4$ & $73 \%$ \\
FRB 121102 Fluxes & 93 & 1.55 & 1.7 & $26 \%$ \\
\hline
\end{tabular}

Table 1. Ratios of most extreme to second most-extreme members of various astronomical datasets. Where meaningful, $N$ is the number of data. Fractional and integral $\gamma$ are theoretical values; of the FRB catalogues, only CHIME contains enough data for a meaningful empirical $\gamma$, which is shown and is close to the theoretical value of $5 / 2$. The final column is the significance of any inconsistency of $x_{1} / x_{2}$ with a single power law with an exponent known either theoretically or empirically from the distribution of less extreme members; lower values imply consistency.

such outliers, attesting to the homogeneity of their populations; even the discovery FRB 010724 is not a significant outlier compared to the second-brightest Parkes FRB 110214. It is no surprise that this analysis confirms that the giant December 27, 2004 outburst of SGR 1806-20 (Hurley et al. 2005; Palmer et al. 2005) is a significant outlier, inconsistent with extrapolation of its lesser flares. Such giant outbursts are naturally explained as a global reordering of a magnetic field much greater than those of radio pulsars, as suggested for the March 5, 1979 outburst of SGR 0525-66 (Katz 1982) while less energetic outbursts may be analogous to Solar flares; this is now known as the "magnetar" model.

The distribution of FRB RM, with $x_{1} / x_{2} \approx 200$, presents a different problem because there is no a priori expectation of the exponent $\gamma$. Eq. 6 indicates that $\gamma>1.43$ can be excluded with $90 \%$ confidence, $\gamma>1.57$ with $95 \%$ confidence and $\gamma>1.87$ with $99 \%$ confidence. In one simple model the rotation measure is predicted to arise from an expanding SNR. In such an object the magnetic field would be expected to scale $B \propto R^{-2} \propto t^{-2}$, the electron density to scale $n_{e} \propto R^{-3} \propto t^{-3}$ and $\mathrm{RM} \propto B n_{e} R \propto R^{-4} \propto t^{-4}$, where $t$ is the age. The irregular fluctuations of the observed RM of FRB 121102 (Hilmarsson et al. 2021) are inconsistent with such a simple model, but it might approximately describe the scaling. If the likelihood of detection does not vary rapidly with time, then the cumulative number $N$ of FRB with ages $\leq t$ is proportional to $t$ and $\gamma=1.25$. Surprisingly, there is not strong statistical evidence that FRB 121102 is an outlier from this model. It would be an outlier from models that indicate a steeper dependence of $N$ on RM. 
Table 1 shows that the homogeneous FRB catalogues are consistent with single power law distributions. The AGN and 3CR (extragalactic) catalogues are not, suggesting some natural scale of activity or bias. A possible source of bias in observations of these variable objects is more frequent observation of the brightest, so they are more likely to be observed in more extreme and luminous states. This is a cautionary tale.

The giant outburst of SGR 1806-20 is definitely inconsistent with an extrapolation of the brightness distribution of its lesser bursts; it is qualitatively different. This is not a new result, but now is quantified. The extraordinary nature of FRB 200428 is hardly a surprise; Galactic objects are much closer than extragalactic objects, and would be expected to be much brighter, an expectation confirmed with high confidence. There is also a less trivial conclusion: the Galaxy does not contain large numbers of micro-FRB, weaker sources of coherent radio radiation than FRB 200428, but still readily observable because of their proximity; FRB 200428 was orders of magnitude above the detection thresholds of the instruments (Bochenek et al. 2020; CHIME/FRB Collaboration 2020) that discovered it. See also the discussions of $\mathrm{Lu}$, Kumar \& Zhang (2020); Margalit et al. (2020). Finally, in at least one model, the extraordinary RM of FRB 121102 is not so extraordinary at all.

\section{DATA AVAILABILITY}

This theoretical study generated no new data.

\section{REFERENCES}

Batchelor, G. 1967 Introduction to Fluid Mechanics (Cambridge U. Press, Cambridge, UK).

Bera, A. \& Chengalur, J. N. 2019 MNRAS 490, L12.

Bennett, A. S. 1962a Mem. RAS 68, 163 vizier.u-strasbg.fr/ viz-bin/VizieR-4 accessed May 25, 2021.

Bennett, A. S. 1962b MNRAS 125, 75.

Bochenek, C. D., Ravi, V., Belov, K. V. et al. 2020 Nature 587, 59.

CHIME/FRB Collaboration 2020 Nature 587, 54.

CHIME/FRB Collaboration 2021 arXiv:2106.04352.

Forman, W., Jones, C., Cominsky, L. et al. 1978 ApJS 38, 357.

Frisch, U. 1995 Turbulence: The Legacy of A. N. Kolmogorov (Cambridge U. Press, Cambridge UK).

Gajjar, V., Siemion, A., Price, D. et al. 2018 ApJ 863, 2.

Göğüus, E., Woods, P. M., Kouvelioutou, C. et al. 2000 ApJ 532, L121.

Golenetskii, S., Apetkar, R., Mazetx, E. et al. 2007 GCN 6775.

Götz, D., Mereghetti, S., Molkov, S. et al. 2006 A\&A 445, 313.

Herta/FRBSTATS 2021 https://ui.adsabs.harvard.edu/abs/ 2021ascl.soft06028S accessed May 25, 2021.

Hilmarsson, G. H., Michilli, D., Spitler, L. G. et al. 2021 ApJ 908, L10.

Hurley, K., Boggs, S. E., Smith, S. M. et al. 2005 Nature 434, 1098.

Katz, J. I. 1982 ApJ 260, 371.

Katz, J. I. 1986 J. Geophys. Res. 91, 10412.

Katz, J. I. 2017 MNRAS 472, L85.

Katz, J. I. 2020 MNRAS 499, 2319

Kolmogorov, A. 1941 Doklady Akademia Nauk SSSR 30, 301.

Kouveliotou, C., Meegan, C. A., Fishman, G. J., Bhat, N. P., Briggs, M. S., Koshut, T. M., Paciesas, W. S. \& Pendelton, G. N. 1993 ApJ 413, L101.
Law, C. J., Abruzzo, M. W., Bassa, C. G. et al. 2017 ApJ 850, 76. Lu, W., Kumar, P. \& Zhang, B. 2020 MNRAS 498, 1397.

Lu, W., Beniamini, P. \& Kumar, P. 2021 ApJ in press arXiv:2107.04059.

Macquart, J.-P. \& Ekers, R. D. 2018 MNRAS 474, 1900.

Margalit, B., Beniamini, P., Sridhar, N. \& Metzger, B. D. 2020 ApJ 899, L27.

Palmer, D. M., Barthelmy, S., Gehrels, N. et al. 2005 Nature 434, 1107.

Petroff, E., Barr, E. D., Jameson, A. et al. 2016 Pub. Ast. Soc. Australia 33, 45 frbcat.org accessed May 31, 2021.

Spinrad, H., Djorgovski, S., Marr, J. \& Aguilar, L. 1985 PASP 97, 932.

Tennekes, H. 1972 A First Course in Turbulence (MIT Press, Cambridge, Mass.).

Wang, F. Y. \& Zhang, G. Q. 2019 ApJ 882, 108.

Wijers, R. A. M. J. \& van Paradijs, J. 1991 A\&A 241, L37.

Zhang, Y. G., Gajjar, V., Foster, G., Siemion, A., Cordes, J., Law, C. \& Wang, Y. 2018 ApJ 866, 149. 